Feb. 3rd. He was improving. The leg was still rigid; he had no power of flexion at the knee; he could walk fairly well, but dragged his leg, trailing the foot along the ground. The toes of this foot were apt to turn inwards, as well as the foot itself, so that he walked then on the outer edge. He felt well in himself-much better. I injected onefiftieth of a grain of atropine subcutaneously.

Feb. 5th. No benefit had resulted from the atropine; it caused marked physiological effects, but did not lessen the rigidity in the least. He could walk better; but his foot still was curved inwards when he walked. In about an hour after I had made this note, I saw him in a severe epileptic attack, in which all his limbs were quite rigid, and he ground his teeth. The dose of bromide of potassium was increased to thirty grains every three hours, and a warm bath ordered.

Feb. 9th. He had no more fits; but, in consequence of a fracas with one of the officials, he wished to go out.

Both these were undoubtedly cases of epilepsy; yet they were not ordinary cases. In the first, the attacks were often much more pro. longed than usual; and indeed it was not always easy to distinguish between the paroxysmal and interparoxysmal disorders. In the attack of March I6th there was prolonged general tremor, but the patient did not lose consciousness at all. So, again, on April 5th, the disorder seems to have consisted solely of giddiness, though it lasted an hour and a half. I do not see how we can refuse to regard these as manifestations of epilepsy, quite as much as the more complete and regular fits which he had previously had. Indeed, if they occurred in a patient who had never had a complete attack, we should feel doubtful as to their exact nature; but in the case before us I can take no other view than that which I have above expressed. And this brings me to a point which I wish to notice on the present occasion; viz., the liability of epilepsy to occur in modes which vary so much from the accepted types, that an inexperienced observer might very easily mistake such cases for instances of some other disease. Unconsciousness, of longer or shorter duration, is one of the most constant phenomena of epilepsy; but it seems quite established that it is not invariably present. Dr. Russell Reynolds, who is not at all inclined to laxity in marking off epilepsy from other disorders, admits among the four modes of attack cases of convulsion having the general form of an ordinary epileptic paroxysm, but in which there is either no loss of consciousness or such slight obscuration as to be at first completely denied by the patient. Such paroxysms may occur at intervals for many years. Dr. Beigel tells us that, out of one hundred and fifty-three cases in which he ob. tained reliable information, one hundred and three had loss of consciousness and general convulsion; thirty had the former without the latter; six had the same with partial convulsion; four had no loss of consciousness, but general convulsion; seven, no loss of consciousness, and no general convulsion; two, no unconsciousness, but partial convulsion; and one, loss of consciousness, and sometimes general, some. times partial, convulsion. Trousseau affirmed that nothing was more irregular than epilepsy in its manifestations, its course, and the fre. quency of its attacks, either in the same or in different patients. $\mathrm{He}$ has had large opportunities of noticing, in his consulting practice, how often the best informed and most enlightened physicians fail to recognise even well marked and grave cases of epileptic disorder, especially of the vertiginous kind (petit mal).

Our second case affords a curious example of one-sided spasm occurring in connexion with epilepsy, and, as I believe, as one of its manifestations. Both pathemata, the tonic spasm of arm and leg, and the epileptic paroxysms, occurred twice at the same periods, which were separated by an interval of seven years. This is certainly strong evidence of their homogeneousness, if I may use the term-of their being both essentially dependent on the same fundamental morbid state. There seems, indeed, to be a wide difference between a sudden violent explosion of convulsion and insensibility occurring at uncertain intervals, and continuous unrelaxing spasm without loss of conscious. ness ; yet it is most certain that the very same causes-viz., irritation of the cerebral membranes from an endostosis, or a tænia in the intestines - may give rise either to continuous giddiness without any convulsion, or to epileptic paroxysms. And, in some cases of epilepsy, giddiness of considerahle duration is a frequent interparoxysmal phenomenon. Again, the same organic lesion may produce a more or less continuous or a strictly periodic neuralgia, the difference of effect being evidently due to some peculiarity in the working of the individual nervous systems. There seems to me, therefore, no great difficulty in comprehending that the same cause might generate both the permanent spasm and the temporary convulsion. Such a case as the one we are reviewing is, however, but an exaggerated instance of the common experience that the interparoxysmal period is occupied by more or less indication of derangement of nervous functions.

The anæsthesia was a remarkable symptom, evidently co-related to the spasm, with which it was in great measure coterminous. I do not know how to explain it, any more than I do the spasm; for of course it is no explanation to say that they both depend on some peculiar state of the motor nervous centres. But it is worth notice, that the two disorders-viz., paralysis and spasm-are constantly found associated together, evidently depending on the same morbid condition; so that it would be quite incorrect to regard them, as you might be inclined to do, as opposites. On account of the spasm being confined to one side -the right-some would be inclined to place the seat of the disorder in the left corpus striatum. On this view, the spasm would be the analogue of the hemiplegic paralysis which occasionally is a sequel of the epileptic paroxysms, and which I suppose to have its seat in the same ganglion. However, as there is no record of any fit having preceded the tonic spasm, and as the latter remained unaffected by the subsequent fits, it must rather be regarded as an independent phenomenon than as a sequel.

The vaso-motor nerves of the right lower limb do not seem to have been involved in the spasm, inasmuch as the temperature of the limb was quite normal. It was curious, however, that the corresponding region of the left was nearly two degrees Fahr. warmer; though, as this statement rests on a single observation, and as it is rather contradicted by a previous one showing that the temperature in buth axillæ was onky 99. 3 deg., I admit that it needs confirmation.

It may well be asked, What was the cause of disorder in this case? And to this question I fear we cannot give a very precise reply. One thing may be affirmed, I am pretty sure; viz., that there was no organic lesion or coarse disease of the nervous centres. Had any such existed, it is tolerably certain that the malady would not have subsided and remained in abeyance for so long a period as seven years. Rather, we may be sure that the symptoms would have become intensified, even if a fatal termination had not ensued. To a certain extent, the recurrence of disorder may be explained by the fact that the patient had been greatly over-exerting his mental faculties for some considerable time before he became ill. The same overstrain has been known to cause delirium, and I have known it produce epileptic attacks; it is, therefore, not unreasonable to regard it as the motor of the disorder in our case. Here, however, as in many other instances, we are obliged to fall back upon some inscrutable peculiarity of the nervous system, which makes the result of the acting of the same cause so different in different individuals. So far, it may be said, the hemispheres in this patient were affected, that he suffered from sleeplessness to a great degree. Translating this into pathological language, we may say that the cerebral exhaustion assumed the form of morbid excitability, as is so commonly the case; and this state did not confine itself to the originally affected centres, but diffused itself to the motor, and in a modified form to the sensory.

The peculiar hunger felt by our patient (which he actually asked me to give him some medicine to quell) was doubtless not a genuine desire for food. I should interpret it as a morbid sensation referred to the gastric nerves, and probably depending on some affection of the solar plexus analogous to that which prevailed in the superior centres.

The treatment indicated in both these cases was evidently calmative and restorative, and was pretty well fulfilled by the remedies we employed. The bromide of potassium answered very well in the first case, but was less decidedly efficacious than I had hoped it would be in the second. I have not yet as much experience of its action in the various kinds of spasm as I hope soon to obtain; but it has not appeared to me so efficacious as I had hoped it would be. This is the experience of others also with regard to this drug in cases of chorea. Of the chloral I need only say that it acted admirably as an hypnotic, as it has in many other cases, but did not materially influence the spasm.

\section{WHAT IS A DIURETIC?*}

BY J. MILNER FOTHERGILL, M.D., Senior Resident Medical Officer of the Leeds Public Dispensary.

A DIURETIC is an agent that increases the flow of urine, is the ordinary answer; and a very incomplete one it is. What do we mean by increasing the flow of urine? Do we mean an increase in its mere bulk - that is, a mere increase of elimination of water-or do we mean an increase of all, or a great part of, the constituents of urine? Certainly we ought to mean the latter, I think. The one action depends to a great extent on the pressure of the circulation merely; the other is a special property of the epithelial cells lining the tubuli uriniferi. It may be desirable to consider each action separately ; firstly, of the pres-

* Read before the Leeds Medical Society, June Ist, 1870. 
sure of the blood within the circulatory organs, or rather arterial ten. sion. The walls of the glomeruli within the Malpighian bodies are excessively thin, and admit of rapid dialysis. Thus, any increase of pressure on the blood on one side causes a rapid passage of its watery constituents to the other : this is the elimination of water. As each drop trickles down the uriniferous tubule it passes along from cell to cell, becoming, in passing, more and more laden with urine excreta. We know well, however, that there is no fixed proportion of action betwixt the amount of water passing in from the glomeruli and the activity of the cells as excretors. We may have a small quantity of urine passed of high specific gravity-as in the decreased urine of advanced cardiac disease, or of profuse perspiration; or we may have a large bulk of urine passed of very low specific gravity; as low as 1.003, or even lower, as in the early stages of granular kidney, or during a hysteric paroxysm. We may then see how, according to different actions, totally different results may follow : we may have a fair elimination of urinary excreta with an accumulating dropsy; we may have a gradually increasing blood-poisoning going on hand in hand with an active excretion of water by the kidneys. Each condition requires its appropriate treatment. It would be as futile in the first condition to administer an agent acting on the renal secreting cells without acting on the circulation and increasing the blood-pressure, as it would be in the other to administer agents which increase the blood-pressure without acting on the secreting cells.

In order to better illustrate this, it may be as well to review the different modes of action in each case and contrast them. In illustration of the effect of increased blood-pressure, take for instance the result of a draught of pure water. If the body be quiet, and there be no unusual activity of skin or bowel-conditions which affect the result as affecting the conditions on which the result depends-there is soon a flow of limpid, odourless urine; there is a rapid absorption of the fluid from the stomach into the blood, increased blood-pressure, and rapid flow in the glomeruli of the kidney. A similar result will follow any great excitement affecting the circulation and increasing the heart's activity, as witness an examination or any similar trying occasion. A like result follows from the action of a hysterical paroxysm. There is an alteration in the blood-pressure again in this condition. There is here a contracted condition of the arteries and violent action of the heart to overcome it. The researches of MM. Cyon, Ludwig, Thiry, and Von Bezold, have demonstrated the connection between the state of the arteries and the palpitation : thus we have, from the opposition of the arteries and the heart's efforts to overcome it, an increased arterial tension, and consequently an increased flow of water from the kidneys, as does actually occur. In a similar manner we have a decided increase of bulk of urine in the earlier stages of granular kidney. The reason of this is intelligible enough. So much of the cortical substance of the kidney is cut off by interstitial inflammation, or increase of connective tissue, and thus their quota is longer added to the acting kidney. Thus there is so much of the secreting substance cut off, but there is no corresponding diminution of the calibre of the renal artery; the pressure in it is unchanged, and thus there must be an abnormal pressure on the portion left intact, and thus increased flow of urine of low specific gravity, as is notoriously the case. Thus we see that the mere bulk of urine depends to a great extent on mere blood-pressure without any increase of vital action on the part of the kidneys themselves.

The second proposition may now be considered. There are agents which act on the renal secreting cells and stimulate them to an increased activity by which an increase in the bulk of urine may be occasioned as well as an increase in the amount of urinary solids. Thus we explain the diuretic action of gin as compared to brandy; the action of buchu as compared to uva ursi ; the action of potash as compared to magnesia; the action of belladonna as contrasted with opium; the action of colchicum as contrasted with digitalis. Substances which act as stimuli to the renal secreting cells and increase their activity, cause them to attract an increased quantity of water by some power which we cannot explain as yet, and so call it vital action. Thus the administration of urea is followed by increased renal action and elimination of it. The kidneys, however, are subject to derangements : thus there may be evidence of great blood-pressure, as in active congestion (Jenner), and the formation of bloody tube-casts, and at the same time a condition of uræmia with a total suppression of urine. Then the decrease of blood-pressure by cupping or purgation is followed by increased renal activity, and soon the flow and elimination of solids may be much over the average on a course of bread-pills, as George Johnson has shown. But here the kidney is in a condition of acute disease. In cases of renal engorgement, we have yet to learn a great deal about the effect exercised by the vasa recta of Virchow and Beale; or, in other words, of the collateral circulation within the kidney itself. Without this safety-valve it is difficult to see how the kidney, with its large afferent artery, could be preserved from utter disorganisation when the convolute circulation is arrested. But without attempting to carry any refinements into the condition of acute renal disturbance, and admitting that it may not yet be possible to explain all the anomalies apparently occurring in these conditions, we may still be able to elucidate, to a great extent the action of external agents on the kidney in a normal, or comparatively normal, condition, and to understand the distinction between increased pressure on the glomeruli and increased action of the renal secreting cells. We can thus appreciate the distinctive difference in the therapeutic agents required in the small and highly dense urine of an enfeebled circulation and the large bulk and low specific gravity of the urine in the early stages of granular kidney. Jenner has told us much about the pressure on the glomeruli, and the distinction, in a diagnostic point of view, between the free flow of primary renal dropsy and the lessened flow where its origin is cardiac. So in the conditions of uræmia or the typhoid condition of fevers, where there is an accumulation of urinary excreta in the blood-the natural stimulus to the kidney secreting cells - the urine may fall off in quantity hand in hand with a growing feebleness of the circulation. There is not sufficient pressure on the glomeruli to produce flow ; and without the washing action of the flow, the secretion of the renal cells cannot be re. moved: here stimulants become diuretics from their action on the circulatory centre. In the hypostatic congestion of typhus, the rolling of the patient over on to one side, and thus mechanically unloading the elevated kidney, is productive of increased diuresis. The dependent condition of the kidneys in the recumbent posture is always a source of danger in enfeebled and acute asthenic conditions. They being placed lower than the general current, a gradual decrease in the bulk of urine passed may be our earliest indication of engorgement of the kidney, and of the danger looming from abolition of renal function; and this may occur when a fair radial pulse may indicate a pretty good general arterial tension.

In the administration of diuretics, too little regard is paid to the manner of action of the agent employed. A mere empirical idea of their asency too frequently prompts their choice, when an accurate knowledge of their special mode of action should determine their selection; and thus the want of success. Without attempting to state that by the selection of agents acting on the circulation, and of others acting on the renal secreting cells, we can produce diuresis with the certainty of mathematics, or forgetting that there are morbid conditions of the kidney where the vicarious action of other organs is the best restorer of renal action, still by a recognition of the different actions, their modes of production, and the means of acting on them, we may practise with greater precision. Thus, in the free flow of granular kidney, some agent which acts on the secreting cells may be required urgently, as potash, colchicum, juniper, or buchu. A real true diuretic may be desirable-indeed, imperative; or even a vicarious action in another organ. Some diuretics have a double action; as, for instance, the scilla maritima. Hilton Fagge and Stevenson found squill to exercise a decided effect on the hearts of frogs, increasing the ventricular contractions in a similar manner to hellebore and digitalis. This, then, is a light as to the diuretic action of squill in asthenic conditions, though no doubt squill is eliminated by the kidneys, as well as the lungs. Belladonna, according to Harley, is also a true double diuretic, being a stimulus to the renal cells, as well as a cardiac tonic; which last it undoubtedly is. The action of gin-that is, alcohol and juniper-combines the two; as does also the spiritus ætheris nitrosi, which, however, acts only slightly on the elimination of urinary solids. Alcohol, ether, and ammonia act by their general stimulant action affecting the circulation. Digitalis is chiefly a diuretic in asthenic cardiac conditions, under which circumstances its action is sometimes magical.

It is, then, no slight matter, the selecting of a diuretic to meet the exact requirements of the patient. The happy-go-lucky choice of past times must be abandoned; and a choice depending on an accurate comprehension of the existing circumstances, and of the action of the agent it is proposed to employ, should take its place. The different conditions of circulation and of the arterial tension must to a great extent guide us in our selection. The effect of position, the portal circulation as affecting the renal circulation, the use and effect of purgation and diaphoresis, must have a value attached to them which a scientific knowledge can alone apportion. The effect of opium and astringents, as tannin, etc., on the-kidney, must not be overlooked. How many men dare not drink port wine, under fear of gout, which will show itself even next day! The action of the renal cells is checked by the astringent, and thus the uric acid is no longer converted by them into urea and carbonic acid; while the stimulus may be producing increased flow from increased pressure. It is of no use, then, in the acute passive congestion of Jenner, to use agents acting on the renal cells, without 
others acting on the blood-tension. It is of no avail to goad the renal secreting cells with stimuli to them in cardiac dropsy, without giving tone to the enfeebled circulation. Thus, when other diuretics have failed, digitalis will usually, in these conditions, produce the expected result. In the removal of accumulations of urinary solids, as after scarlatinal dropsy, etc., plain water may be a most effective agent, and be followed by increased flow of urine of fair specific gravity. We must understand clearly what we mean by a diuretic, and what we propose to accomplish, before determining upon the use of any agent or combination of agents, if we wish our efforts to be successful. And the choice of diuretics cannot be a mere matter of toss-up by any one who aspires to the character of a scientific practitioner, or of being capable of giving a reason for the faith that is in him.

\section{ON THE TREATMENT OF SCARLATINA AND OF SCARLATINAL DROPSY.*}

By RICHARD C. SHETTLE, M. D., Physician to the Royal Berkshire Hospital, Reading.

Ar the last meeting of this Society, some cases were related in which epileptiform convulsions attacked individuals suffering from dropsy after scarlatina; the convulsions and dropsy yielded to hot air baths and active purgation by means of compound jalap powder; but, after a slight interval of freedom from anything abnormal, sudden and fatal congestion of the lungs supervened.

A case having recently come under my own observation, of an identical character, so far as the epileptic convulsions and dropsy, but which yielded to treatment of a different nature, I considered I was only doing my duty as a member of this Society in making mention of it. Thereupon I was requested to bring the matter forward for discussion at the next metting, and, as it was considered that the line of practice which I advocated in scarlatina was somewhat unusual, to state the principles of that practice and the grounds upon which I based it. I have much pleasure in acceding to the request.

The notes of the case in question have been kindly forwarded to me by $\mathrm{Mr}$. Moore, in conjunction with whom I attended the patient. They are as follows. "My attendance had ceased on 27th November, as Mr. L. was considered well from an attack of scarlatina. On December 3 rd, at 5 p.m., I was sent for, as he had severe headache, and said he must go mad. I gave him one grain of opium, which was rejected; I gave him another grain at 9 p.m., and he went to sleep, and slept till 3 a.m., when his mother found him tossing his arms about; he said he thought he was going to have a fit; he went to sleep again, and, at 5 a.m. he had a violent epileptic fit. I was sent for at that time, viz., 5 a.m., December 4 th. He continued to have these fits every hour until one o'clock, and then he had two in one hour. Dr. Shettle came about 2 p.m., and prescribed as follows. B Argenti nitratis gr. iss acid. nitrici diluti $3 \mathrm{j}$; potassæ nitrat. $3 \mathrm{j}$; aquæ dest. $\zeta$ vi. A sixth part to be taken every hour. Two hot mustard baths were also administered. Mr. L. had no fit after Dr. Shettle came; and the urine, which was highly albuminous, after two days became free from albumen, and in a fortnight's time he went to the Isle of Wight, and has remained well since."

It is now, I believe, universally considered that scarlatina is absolutely due to blood-poisoning, and all the premonitory symptoms may be said to indicate exhaustion or malnutrition of the nervous system. but superadded to the general symptoms we have others, such as the throat affection and the rash on the skin.

The throat affection, which varies in intensity in accordance with the severity of the attack, is very characteristic of the channel by means of which the virus enters the system; and it appears to me that the lecture lately delivered by Professor Tyndall at the Royal Institution pretty closely establishes this point. To use the Professor's own words : "In all probability the protection of the lungs will be the protection of the entire system; for it is exceedingly probable that the germs which lodge in the air-passages and which at their leisure can work their way across the mucous membrane, are those which sow in the body epidemic disease."

Now, as under healthy respiration oxygen is attracted from the air, and absorbed by the corpuscles of the blood, and is then carried by those corpuscles to the tissues of the body, to impart life and heat, so, also, must these germs of organic matter be absorbed and carried away by the same means; but, instead of adding to the vitality, they would, according to the proof afforded by Pasteur, give rise to a process of fermentation, by finding in the corpuscles the necessary food for their

\footnotetext{
* Read before the Reading Pathological Society.
}

support. The result of such a fermentative process on the corpuscles primarily, and the whole body secondarily, would be, first, the increased development of heat, and, secondly, the impairment of vitality, because that vitality would be more or less destroyed in supporting the parasitic growth. These effects would be modified, first, by the nature or peculiar character of the organic particles, and, secondly, by the extent to which the atmosphere was charged with them, and also by the degree of health or vigour in which they found the corpuscles to which they attached themselves. If this opinion be correct, we at once establish a cause for the increase of temperature in fevers generally, as they doubtless have a common origin, and also for the depression of the vital powers. In scarlatina, the blood-poisoning appears to be wellmarked from the commencement in all stages and degrees of the complaint ; and, such being the case, the most successful treatment must depend upon the most rapid and safe method of destroying or eliminating the virus ; and, whatever means we use to facilitate this process, the skin or kidneys must be great channels for its removal. The skin, however, from the amount of action which takes place in it under an ordinary attack of scarlatina, becomes almost useless for the purpose : whilst the kidneys are frequently altogether prevented from performing their functions from the congested state in which they exist. How then shall we best remedy the evil? I reply, by assisting nature to effect her own cures : first, by removing the dried cuticle, by means of diaphoretics, warm baths, and friction, so as to enable the skin to do its work; secondly, by relieving the congested state of the kidneys; thirdly, by giving tone or power to the nervous system for the due performance of its accustomed duty; for it would be of but very little service to use means for the relief of the skin or kidneys if those means were not calculated to raise the powers of life, already more or less impaired. Thus, a bath administered for too long a time, or of too high temperature, would diminish the vital powers ; nay, more, it might so depress as to cause death; whilst active purging by compound jalap powder, or remeadies of the same class, might, and I believe often does, cause irremediable mischief. By such means, it is true, a large quantity of fluid may be carried out of the circulation with considerable rapidity; and so that state of congestion of the kidneys which gives rise to dropsy would be relieved ; but the ultimate issue of a case so treated would de. pend materially, if not entirely, upon the degree of reparative power which the individual was capable of exerting. If the powers of life were strong, speedy and permanent relief might be afforded; but if these powers were already considerably exhausted, there can be no question that active purging or depletion of any kind would either destroy life, or so materially depress, that the temporary relief would speedily be followed by a much greater amount of depression, and congestion of some one or more of the internal organs. Indeed, nothing is more common than the occurrence of passive congestion in diseases attended with great and sudden prostration, as is the case in fevers of a typhoid or malignant character. It is also well known that the operation of evacuant medicines may produce the same result.

After what I have already pointed out as the chief points to be observed in the treatment of scarlatina, I think it will be readily understood that, whilst on the one hand I should support the system by means of beef-tea and other articles of diet that can be readily conveyed into the blood, I should, on the other, avoid as much as possible alcoholic stimulants : first, because to benefit the patient by their administration we must continue their use until the disease had in great measure subsided, or the depression which invariably follows their exhibition would be a source of mischief; secondly, because I believe that, with but few exceptions, they may be supplanted by remedies which act almost as immediately upon the nervous system, and of which the stimulating or tonic effects are more persistent. The nitrate of silver is one of these; and, indeed, I believe there are few remedies of this. class that act so rapidly, so energetically, and so persistently as this does.

As a form of combination for the treatment of scarlatina, I may say that that prescribed in the case referred to is generally adopted by me; and as to the modus operandi, I may express my belief that the nitrate of silver acts directly on the peripheral nerves, and so stimulates the whole nervous system, whilst the nitric acid gives tone to the blood (it is known to promote the formation of fibrine), and especially strengthens the kidneys; and the nitrate of potash promotes the secretion of urine and rapid elimination of the virus of the disease. In any case of scarlatina, I am of opinion that we cannot begin to assist nature too soon, by means of baths, with or without mustard, the internal re medies to which I have just alluded, and such others as specialities of cases may render necessary. The throat-affection is, I believe, best met by administering sulphurous acid spray, and at the same time the system must be well supported with food. Were such treatment adopted, I believe we should hear much less of scarlatina dropsy or any of the other 